\title{
An Interesting Intraoperative Decision
}

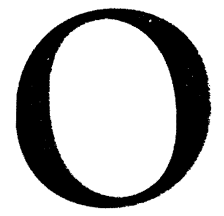

NE NIGHT in March 1984 , we were in the process of removing a donor liver for transplantation from a young trauma victim. After some dissection in the porta hepatis, we learned that the potential recipient had an electrocardiogram on admission that showed recent myocardial injury. Because the recipient did not have terminal liver disease, we attempted to place the liver at another center rather than risk further myocardial injury to the patient during the operation. There was no national system of organ sharing at this time, and placement of organs was usually done on an ad hoc basis from surgeon to surgeon. Our attempts in this case were unsuccessful. Having completed most of the dissection in the porta hepatis, we elected to shift gears and transplant the pancreas instead.

1 had just returned from Pittsburgh, Pa, where I had read a preprint of the article by Starzl et $\mathrm{al}^{1}$ on whole organ pancreas transplantation. A few days earlier we had evaluated a 47-year-old man with diabetes and renal failure who lived only a few blocks from the hospital. Although he had extensive coronary artery disease, we thought that a simultaneous pancreas and kidney transplantation might be his best option. He had no preformed antibody that obviated the necessity for a preliminary cytotoxic crossmatch. Shortly after the telephone call, he arrived in the operating room. A workup for kidney transplantation had been completed a few days earlier.

We proceeded to complete the donor operation, removing the whole pancreas along with the kidneys instead of the liver. No "back table" preparation of either organ was necessary because the entire dis- section was performed in situ. Starzl et al ${ }^{1}$ had already shown that a short duodenal "bubble," a few centimeters in length, could be transplanted safely, which avoided surgical complications associated with transplantation of a longer segment of small intestine. We also transplanted the spleen to provide a more physiologic blood flow through the organ.

The patient became normoglycemic, and except for developing graft vs host disease, which required removal of the donor spleen, had a benign postoperative course. He did not show signs of rejection for the next 3 months and was normoglycemic. but had a myocardial infarction while riding a roller coaster a few months after undergoing transplantation. He eventually died of the infarction but had normal renal and pancreatic islet function.

In the next 15 months we performed 20 of these procedures with moderate success. Two patients died within the first year, the kidneys functioned in 17 , and the pancreas in 15 at the time of publication of the series. ${ }^{2}$

Although the technique of pancreas transplantation evolved in the ensuing years, including the bladder drainage technique introduced by Nghiem et al, ${ }^{3}$ we have now come full circle and have adopted the enteric drainage procedure, ${ }^{4}$ which avoids the high complication rate associated with duodenocystostomy. Bladder drainage is normally reserved for solitary pancreas transplantation (without simultaneous transplantation of the donor kidney), which permits measurement of the urinary amylase, a decrement of which signals possible rejection.

Today, transplantation of the pancreas with or without the kidney is an accepted therapeutic procedure for patients with type 1 dia- betes. Results in our center and others have limited mortality and graft success rates that exceed 95\% and $85 \%$ for the kidney and pancreas, respectively. Starzl's pioneering work in the early 1990 s with tacrolimus, as well as his contributions in developing the operative technique in the early 1980 s, paved the way for pancreas transplantation. Sollinger et $\mathrm{al}^{5}$ stated at a meeting of the American Surgical Association that pancreas transplantation is has the highest long-term sucess rate of all cadaver organs transplanted.

Perhaps a quotation by Goethe is appropriate. "Whatever you can do, or dream you can do, begin it. Boldness has genius, power, and magic in it."

$$
\begin{aligned}
& \text { Robert J. Corry, MD } \\
& \text { Thomas E. Starzl } \\
& \text { Transplantation Institute } \\
& \text { University of Pittsburgh } \\
& \text { Medical Center } \\
& \text { 3601 Fifth Ave } \\
& \text { Fourth Floor Falk Clinic } \\
& \text { Pittsburgh, PA 15213 } \\
& \text { (e-mail: roadwayla@ } \\
& \text { msx.upmc.edu) }
\end{aligned}
$$

\section{REFIRINCIS}

1. Starzl TE, Iwatsuki S, Shaw BE, et al. Pancreaticoduodenal transplantation in humans. Surg Gynecol Obstet. 1984;159:265.

2. Corry RJ, Nghiem DD, Schulak JA, Beutel WD, Gowna TA Surgical treatment of diabetic nephropathy with simultaneous pancreatic duodenal and renal transplantation. Surg Gynecol Obstet. 1986;162:547-555

3. Nghiem DD, Beutel WD, Corry RJ. Duodenocystostomy for exocrine pancreatic drainage in experimental and clinical pancreaticoduodenal transplantation. Transplant Proc. 1986;18:1762-1764.

4. Corry RJ, Egidi MF, Shapiro R, et al. Pancreas transplantation with enteric drainage under tacrolimus induction therayy. Transplant Proc. 1997; 29:642.

5. Sollinger HW, Odorico JS, Knechtile SJ, et al. Experience with 500 simultaneous pancreaskidney transplants. In: Abstract book of the AmeriCan Surpical Association; Aprit, 2-8, 1998. 